American Journal of Pharmaceutical Education 2020; 84 (4) Article 7453.

\title{
BRIEF
}

\section{Pharmacy Students' Ability to Identify the Steps of the Pharmacists' Patient Care Process During IPPE}

\author{
Stacy Taylor, PharmD, MHA, ${ }^{\mathrm{a}}$ Erin Deja, PharmD, ${ }^{\mathrm{b}}$ Holly Divine, PharmD, ${ }^{\mathrm{a}}$ \\ Lyndi Laney, PharmD, MBA, ${ }^{c}$ Tera McIntosh, PharmD ${ }^{\mathrm{a}}$ \\ ${ }^{a}$ University of Kentucky, College of Pharmacy, Lexington, Kentucky \\ ${ }^{\mathrm{b}}$ Moses H. Cone Memorial Hospital, Greensboro, North Carolina \\ ${ }^{\mathrm{c}}$ BayCare Health System, Clearwater, Florida \\ Submitted November 7, 2018; accepted September 3, 2019; published April 2020.
}

Objective. To determine the ability of first-year Doctor of Pharmacy (PharmD) students to describe patient care activities performed by pharmacists and accurately associate those activities with the five core steps of the Pharmacists' Patient Care Process (PPCP).

Methods. First-year student pharmacists completed introductory practice experiences at ambulatory and institutional pharmacies. Students' ability to describe activities that occurred in these settings and align them with the five core steps (collect, assess, plan, implement, and follow-up) of the PPCP were assessed.

Results. The students were more adept in describing patient care activities and aligning them to the appropriate PPCP steps in an ambulatory pharmacy setting than in an institutional pharmacy setting. Students achieved higher scores when describing and aligning patient care activities associated with the collect and implement steps in an ambulatory pharmacy setting and for the assess step in an institutional pharmacy setting.

Conclusion. As institutions strive to adopt the PPCP in all areas of the curriculum, this application serves as an example of successful PPCP integration early in the introductory experiential curriculum. Our results challenge faculty to provide early opportunities for students to apply the PPCP in a variety of practice settings, including settings focused on the medication distribution system.

Keywords: pharmacists' patient care process, introductory pharmacy practice experience, experiential education

\section{INTRODUCTION}

The Pharmacists' Patient Care Process (PPCP) was developed by the Joint Commission of Pharmacy Practitioners to promote a consistent approach to delivery of patient-centered care across the profession of pharmacy. ${ }^{1}$ Use of the PPCP is intended to be universally applicable to pharmacists' provision of patient care in any practice setting. Recognizing the importance and potential positive impact a standard process could have on the profession, the Accreditation Council for Pharmacy Education (ACPE) incorporated the PPCP into Standards 2016, requiring pharmacy curricula to prepare students to provide care according to the PPCP. ${ }^{2}$ The American Association of Colleges of Pharmacy (AACP) additionally underscored the value of widespread dissemination of the PPCP by charging three workgroups with designing strategies to

Corresponding Author: Stacy Taylor, University of Kentucky, College of Pharmacy, 789 S. Limestone St., Lexington, KY 40508. Tel: 859-323-5046. Email:

stacy.taylor@uky.edu accelerate the adoption of the PPCP in pharmacy curricula. $^{3}$ The workgroups developed guidance for schools, emphasizing the value of integrating the PPCP into classroom, simulated, and experiential learning activities and across the assessment continuum. ${ }^{3}$

Because the PPCP is a relatively new process, schools and colleges of pharmacy are beginning to report their experiences of integrating the PPCP into curricula. Early reports describe PPCP integration primarily in pharmacotherapy courses with pedagogy consisting of lecture presentations, assigned readings, incorporation into patient cases, and homework assignments. ${ }^{4-6}$ Assessment of PPCP learning outcomes in these applications consists of patient care notes and examination questions mapped to the PPCP. Several schools have also provided early descriptions via AACP posters, newsletters, and commentaries on the incorporation of the PPCP in simulated patient care laboratory sessions $s^{7,8}$ and introductory and advanced experiential education. ${ }^{9-11}$ One manuscript published in the Journal described student performance using the PPCP in a capstone course taught immediately 


\section{American Journal of Pharmaceutical Education 2020; 84 (4) Article 7453.}

prior to advanced pharmacy practice experiences (APPEs) ${ }^{12}$; however, to our knowledge, there are no published data on student learning outcomes subsequent to implementing PPCP assignments in introductory pharmacy practice experiences (IPPEs).

Contemporaneous to the development and publication of the PPCP, the University of Kentucky College of Pharmacy (UKCOP) implemented a new Doctor of Pharmacy (PharmD) curriculum. One of the central tenets when in design of the new curriculum was that the PPCP should be intentionally integrated throughout the curriculum from professional year 1 (P1) to professional year 4 (P4), in classroom, simulated, and experiential coursework. The PPCP is first introduced via lecture and flipped classroom activities in two first-semester P1 courses: Transitions in Pharmacy and Clinical Reasoning. Students then have multiple opportunities to practice aspects of the PPCP in a re-engineered patient care laboratory/ experiential course sequence, entitled Patient-centered Care Experience, which exposes students to ambulatory practice, institutional practice, and patient assessment skills. In this six-semester course sequence, student pharmacists progress from employing the PPCP in cases in which the patient has a single medication therapy problem during the P1 year, to cases in which the patient has multiple acute and chronic medical conditions and more complex medication regimens by the end of the P3 year. The Patient-Centered Care Experience is also designed to encompass a complementary element of experiential coursework or "fieldwork" each semester in an ambulatory or institutional setting that evolves into longitudinal IPPEs as the curriculum progresses. In the fall, P1 students enrolled in the course begin applying the PPCP in activities such as taking medication histories, medication counseling, and providing self-care consults during the first four weeks of the semester. Students are also learning about medication distribution processes early in the semester; therefore, $\mathrm{P} 1$ fieldwork in the fall semester is designed to afford students exposure to the use of the PPCP in medication distribution systems in both ambulatory and institutional settings. The intent of these experiences is to allow early learners to observe how the knowledge and skills they are acquiring in the classroom and simulated coursework are applied and operationalized in real-world pharmacy practice and to provide context for the content being taught in the didactic portion of the curriculum.

The primary objective of this research was to determine early student pharmacists' ability to describe patient care activities performed by pharmacists within ambulatory and institutional medication distribution systems and accurately align those activities to the five core steps (collect, assess, plan, implement, and follow-up) of the
PPCP. ${ }^{1}$ Additionally, differences were examined in student ability to describe and align the steps of the PPCP in ambulatory vs institutional settings.

\section{METHODS}

This research was approved by the University of Kentucky Institutional Review Board. Eligible study participants included 139 P1 student pharmacists enrolled in the Patient-Centered Care Experience course in fall 2016, all of whom visited ambulatory and institutional pharmacy practice sites for one hour each to complete a guided educational experience focused on the medication distribution system. Timing of the visits were scheduled to occur concurrently with their ambulatory and institutional modules in the course. Students visited the ambulatory sites in pairs in the middle of the semester and visited the institutional sites in groups of four to five students during the latter portion of the semester. Student group sizes were established based on requests of the pharmacist preceptors according to capacity in each setting. Each student was assigned to one of four ambulatory care sites affiliated with the academic medical center campus, and all students were assigned to a single institutional site, the central pharmacy of an academic medical center. Experiential faculty members collaborated with preceptors at the pharmacies to develop a facilitators' guide to standardize the experience for each student team. For the ambulatory sites, pharmacists who regularly worked at the four facilities served as preceptors for the activity, providing a cumulative total of 70 hours of precepting time. As part of their small-group teaching requirement, pharmacy residents guided groups of students through the institutional experience over a four-day period for a total of 32 hours of teaching time between the two residents.

One of the learning objectives for the fieldwork experience was to describe patient care activities occurring in the medication distribution system and align those activities to the correct steps of the PPCP. One week following their assigned fieldwork, students submitted two complementary assignments, one for ambulatory and one for institutional, specifically designed to assess this learning objective. Students were instructed to describe the patient care activities of the pharmacist occurring within the medication distribution system, and then to label each patient care activity according to its corresponding PPCP step. The steps of the PPCP were listed at the bottom of the assignment to aid students in completing this task. As components of the fieldwork activity, students also completed a written reflection and participated in a required in-person small-group debriefing session at the end of the semester. For the debriefing session, students were divided into 16 groups of eight or nine students 


\section{American Journal of Pharmaceutical Education 2020; 84 (4) Article 7453.}

each. Fifty percent of the small groups were facilitated by faculty members, $25 \%$ by residents, and $25 \%$ by APPE students. Each facilitator used a standard list of debriefing questions to guide the discussion. In the debriefing session, students discussed each step of the PPCP in the context of the medication distribution system, recalling specific patient care activities they observed occurring at various points and how those aligned to the PPCP.

Using the list of patient care activities enumerated in the PPCP, students' ambulatory and institutional assignments were examined to determine whether students described activities corresponding to each of the five steps and correctly aligned the activity to the appropriate core step. For each step in the PPCP, students earned one point for describing one or multiple patient care activities on the list and earned an additional point for correctly aligning that patient care activity to the appropriate PPCP step. This yielded a possible score of two points for each step of the PPCP. Because there are five steps in the PPCP, the maximum score for each of the ambulatory and institutional assignments was 10 points. The scoring method was tested on a random sample of five ambulatory and institutional assignments. In an effort to decrease ambiguity and potential variability between graders, further discussion ensued regarding how to score vague responses or technical tasks that were described as patient care activities. The Entrustable Professional Activities (EPAs) ${ }^{13}$ were referenced to delineate patient care activities from related technical tasks in the medication use system. We determined that written descriptions needed to provide evidence that the student recognized intentional cognitive actions performed by the pharmacist. For example, students received one point for describing a patient care activity that represented the collect step of the PPCP, ie, "the pharmacist uses resources such as the patient, caregiver, patient profile, or electronic medical record to gather pertinent information." Students then received one additional point if they correctly aligned this activity to the collect step. On the other hand, students did not receive credit for a identifying a patient care activity representing the collect step if they simply reported tasks such as "refilled a prescription over the phone," "scanned a prescription into computer," or generically reported "pharmacists received orders." If they did not report a suitable patient care activity, then they were ineligible to receive the second point for aligning the activity with the collect step. Similarly, with regard to the assessment step, students who reported "pharmacist performs final product check, ensuring medication, dose, and frequency are appropriate for the patient's diagnosis and renal function" received credit for describing an assessment patient care activity and were eligible to earn one additional point if they correctly aligned this activity to the assessment step. However, answers such as "pharmacist performs final product check to ensure correct patient, correct directions, and correct medication has been prepared" did not receive credit for identifying patient care activity representing the assessment step of the PPCP and were not eligible to receive the alignment point. The iterative development of the scoring process occurred over a series of several meetings involving three experiential faculty members and two advanced pharmacy practice experience students who were completing an academic APPE. Once the scoring process was finalized, submissions previously reviewed were re-scored.

Of the 139 students in the course, 122 consented to permit use of their de-identified data for research purposes. Two students completing an academic APPE served as scorers and independently graded a random sample of 30 students' paired ambulatory and institutional assignments. Grading discrepancies were reconciled, with an experiential faculty member serving as arbitrator in cases of disagreement. The scoring process for each student's assignment took approximately one hour. Using this preliminary sample of 30 paired assignments, we performed a power and sample size study using JMP 12 (SAS, Cary, NC). We determined that a random sample of 58 students would detect a difference of 0.75 points in the total assignment score between ambulatory and institutional assignments. Based on this analysis, a sample of 61 paired ambulatory and institutional assignments were assessed (50\% of eligible students) using the same process described above with two independent scorers and one arbitrator to resolve cases of disagreement.

Statistical analyses were performed using SPSS Statistics, version 23 (IBM, Armonk, NY). A paired samples $t$ test was used to compare the overall score earned for the ambulatory vs the institutional reflective assignment. The related-samples Wilcoxon signed rank test was used to compare the score earned for each step of the PPCP for the ambulatory vs the institutional assignment. A one-way ANOVA was used to test for differences between ambulatory care sites. An alpha level of .05 was used to determine significance.

\section{RESULTS}

Sixty-one paired ambulatory and institutional reflective assignments were graded to determine student pharmacists' understanding of the pharmacists' role in the PPCP. Mean scores for the overall assignment and for each individual step of the PPCP are displayed in Table 1. The mean scores for ambulatory and institutional assignments were 5.1 and 4.0 out of $10(p<.01)$, respectively. No differences were found in mean scores based on 


\section{American Journal of Pharmaceutical Education 2020; 84 (4) Article 7453.}

Table 1. Ability of Students Enrolled in an Introductory Pharmacy Practice Experience to Correctly Identify the Steps of the Pharmacists' Patient Care Process in Actual Practice Settings $(\mathrm{N}=61)^{\mathrm{a}}$

\begin{tabular}{lccc}
\hline Scores & Ambulatory Assignment, Mean (SD) & Institutional Assignment, Mean (SD) & $p$ Value \\
\hline $\begin{array}{l}\text { Total score } \\
\text { PPCP steps }\end{array}$ & $5.1(2.5)$ & $4.0(2.1)$ & $<.01^{\mathrm{b}}$ \\
Collect & & & $.03^{\mathrm{b}}$ \\
Assess & $1.0(1.0)$ & $0.7(0.9)$ & $<.01^{\mathrm{b}}$ \\
Plan & $1.3(0.9)$ & $1.8(0.6)$ & .76 \\
Implement & $1.0(0.9)$ & $1.0(0.8)$ & $<.01^{\mathrm{b}}$ \\
Follow-up & $1.4(0.7)$ & $0.3(0.7)$ & .07 \\
\hline
\end{tabular}

Abbreviations: PPCP $=$ Pharmacists' Patient Care Process

${ }^{\text {a }}$ Total score out of 10 points; other steps out of 2 points

${ }^{\mathrm{b}} p<.05$ was considered significant

ambulatory care site visited $(p=.40)$. When comparing student ability to correctly describe and align patient care activities associated with the individual steps of the PPCP in ambulatory and institutional pharmacies, students scored higher for the collect $(p=.03)$ and implement steps $(p<.01)$ on the ambulatory assignment and higher for the assess step $(p<.01)$ on the institutional assignment.

As shown in Table 2, students scored highest on the ambulatory assignment for describing patient care activities associated with the implement step, with $89 \%$ $(54 / 61)$ of students successfully describing instances when ambulatory pharmacists implemented patient care. The most commonly reported ambulatory patient care activity for implement was "counseling the patient." When evaluating the institutional assignment, students demonstrated the most competence in describing patient care activities associated with the assess step, with $93 \%$ (57/61) correctly identifying instances when pharmacists assessed and analyzed patient information. The most commonly cited institutional activities associated with the assess step included "evaluating the medication order for appropriateness" and "pharmacists verifying orders." Describing instances of follow-up were low in both the ambulatory and institutional assignments, with only $23 \%$ $(14 / 61)$ and $10 \%(6 / 61)$ of students listing patient care activities associated with the follow-up step for each assignment, respectively. In regard to the institutional assignment, $23 \%$ of students correctly listed patient care activities associated with the implement step. Instead of listing PPCP implementation patient care activities in the institutional setting, students often incorrectly listed technical tasks such as "filling, compounding, labeling, and delivering medications" as implementation steps. When examining the percent of students who correctly aligned the patient care activities they described, students were able to align activities to the correct step of the PPCP with two exceptions. For both the ambulatory and institutional assignments, students had difficulty correctly aligning patient care activities to the plan and implement steps, with only $61 \%$ and $50 \%$ of activities correctly aligned with these steps, respectively.

\section{DISCUSSION}

This study highlights student learning outcomes of an early application of the PPCP in the experiential curriculum. Overall, student ability to describe patient care activities and align them to the appropriate core PPCP steps was only demonstrated with $51 \%$ accuracy in an ambulatory pharmacy setting and $40 \%$ accuracy in an institutional pharmacy setting. Students were more successful

Table 2. Ability of Students Enrolled in an Introductory Pharmacy Practice Experience to Describe Patient Care Activities and Align them to the Steps of the Pharmacists' Patient Care Process on Ambulatory and Institutional Assignments $(\mathrm{N}=61)$

\begin{tabular}{|c|c|c|c|c|}
\hline \multirow[b]{2}{*}{ PPCP Step } & \multicolumn{2}{|c|}{ Ambulatory Assignment } & \multicolumn{2}{|c|}{ Institutional Assignment } \\
\hline & Described, No. (\%) & Aligned, ${ }^{\mathrm{a}}$ No. $(\%)$ & Described, No. (\%) & Aligned, ${ }^{\mathrm{a}}$ No. $(\%)$ \\
\hline Collect & $33(54)$ & $30(91)$ & $26(43)$ & $18(69)$ \\
\hline Assess & $42(69)$ & $34(81)$ & $57(93)$ & $51(90)$ \\
\hline Plan & $36(59)$ & $22(61)$ & $40(66)$ & $20(50)$ \\
\hline Implement & $54(89)$ & $33(61)$ & $14(23)$ & $7(50)$ \\
\hline Follow-up & $14(23)$ & $13(93)$ & $6(10)$ & $6(100)$ \\
\hline
\end{tabular}

Abbreviation: PPCP $=$ Pharmacist Patient Care Process

${ }^{\text {a }}$ Percent calculated for only those students who accurately described the PPCP step 


\section{American Journal of Pharmaceutical Education 2020; 84 (4) Article 7453.}

in describing and aligning the PPCP steps with activities that occurred in an ambulatory pharmacy than with those that occurred in an institutional pharmacy. Student ability to describe and correctly align pharmacist patient care activities also varied for the individual steps of the PPCP. Students scored higher in describing and aligning patient care activities associated with the collect and implement steps in an ambulatory pharmacy and achieved higher scores for the assess step in an institutional pharmacy.

The AACP is dedicated to developing strategies to accelerate the adoption of the PPCP in pharmacy curricula and has emphasized the value of integrating the PPCP in all areas of the curriculum including classroom learning, simulated patient care laboratories, experiential settings, and curricular assessment. ${ }^{3}$ Our study provided an early opportunity for students to apply the PPCP in two practice settings focused on the medication distribution system. First-year student pharmacists were only able to describe patient care activities and align them to the core steps of the PPCP with approximately $45 \%$ accuracy. One possible explanation is the students' level of training at the time of the study and lack of familiarity with the cognitive patient care processes required in medication distribution. Student performance on this assignment can serve as a baseline measure of student knowledge of the PPCP. Our findings support providing students with continued practice opportunities to improve their ability to recognize and align patient care activities to the PPCP. Learning to describe patient care activities that correspond to each step of the PPCP will provide a process framework preparing student pharmacists for full engagement in the PPCP at later points in the experiential curriculum.

The students may have more often recognized the collect and implement steps in the ambulatory setting because pharmacists often complete these steps directly with patients in the pharmacy. During prescription intake, ambulatory pharmacists directly gather information from the patient, whereas in inpatient settings pharmacists are usually collecting information about the patient from various sections of the medical record. Although students observed inpatient pharmacists gathering information from various areas of the medical chart during order verification, it may have been more difficult for early students to conceptualize this as a collect step. Similarly, patient counseling was frequently listed for the implement step and is a highly visible patient care service in an ambulatory pharmacy. While patient counseling certainly occurs in an inpatient setting, it would not have been observed directly by students participating in this experience occurring within an institutional pharmacy. Within the inpatient experience, students had the opportunity to observe pharmacists modifying orders, but very few students described this as a patient care activity. Once again, this was possibly because of the difficulty an early learner may have in conceptualizing this as an implement step. When further considering reasons for the difference in student outcomes, taking a medication history and patient counseling are among the first skills students are taught and able to practice in the first semester in the PatientCentered Care Experience course. This may have made it easier for P1 pharmacy students to recognize and map the collect and implement steps.

As for students' stronger performance on the assess step in the institutional setting, the cognitive process may have received greater or more consistent emphasis in the inpatient experience. While most new pharmacy students have previously been inside ambulatory pharmacies prior to pharmacy school, a much smaller proportion have been inside institutional pharmacies. During the inpatient experience, prior to students sitting down with the pharmacist processing orders, the pharmacy resident provided some examples of what institutional pharmacists evaluate, analyze, and consider when verifying orders. Consistently stating the cognitive processes that occurred in this area of the pharmacy likely contributed to students correctly identifying the assess step in the institutional assignment. While these same cognitive processes are required in an ambulatory pharmacy setting, limited anecdotal student feedback raised concerns that ambulatory preceptors may have failed to consistently explain to students their cognitive considerations when processing prescriptions. The differences observed in student performance on this item reinforce the need to ensure that preceptors in ambulatory care settings consistently cover this area and not assume that student familiarity with ambulatory pharmacies translates into student understanding of the cognitive processes required for prescription verification.

In both settings, students were least skilled in describing and aligning follow-up or monitoring patient care activities. This step of the PPCP may occur less frequently or be less visible in the ambulatory pharmacy setting during the medication distribution process that was showcased as part of this guided experience. Patient follow-up and monitoring are readily observable in the ambulatory pharmacy during medication therapy management and self-care consults. However, because student time for this experience was limited to one hour, many students may not have had an opportunity to observe the follow-up step of the PPCP. In the institutional pharmacy that the students visited, pharmacy services are largely decentralized, with follow-up and monitoring being a primary responsibility of the pharmacists working on the floors. While pharmacists in central pharmacy also 


\section{American Journal of Pharmaceutical Education 2020; 84 (4) Article 7453.}

complete follow-up activities when reviewing an order to change an existing drug, dose, or frequency, early students may not have recognized the monitoring aspect that is occurring as part of order review.

There were several limitations to this study. One was that all students visited the ambulatory pharmacies midsemester and then the institutional pharmacy later in the semester. It is unknown whether this sequencing, other coursework, or some students' prior work experience may have had an impact on the student performance and outcomes we observed. Additionally, the ratio of students to preceptors was two to one in the ambulatory pharmacies and four or five to one in the institutional pharmacy. This may have led to differences in student performance on the two assignments. Another item that may have influenced student outcomes related to the pharmacy resident and preceptor training for the experience, which primarily focused on providing a consistent portrayal of facets of the medication distribution system to students and did not provide specific training on the PPCP. The following year, the facilitator guide and preceptor training were amended to include specific training on the PPCP and included instructions to consistently explain the pharmacists' cognitive processes and patient care activities that occur at various points in the medication distribution system.

To increase the generalizability of the PPCP, further implementation and study are needed. Although it appears that student comprehension of the PPCP can be assessed in an early experiential activity, the results we provide are from a single institution and a single cohort of students. In the future, it will be important to evaluate students' ability to apply the PPCP in a wider variety of introductory experiential settings. Finally, it would also be interesting to follow student learning outcomes as they progress through the experiential curricula.

\section{CONCLUSION}

As institutions strive to adopt the PPCP in all areas of the curriculum, this application serves as an example of successful integration early in the introductory experiential curriculum. Our results challenge faculty members to provide early opportunities for students to apply the PPCP in a variety of practice settings, including those focused on the medication distribution system.

\section{ACKNOWLEDGMENTS}

The authors acknowledge Aric Schadler for his statistical support on this project.

\section{REFERENCES}

1. Joint Commission of Pharmacy Practitioners. Pharmacists' Patient Care Process. May 29, 2014. http://www.pharmacist.com/sites/ default/files/files/PatientCareProcess.pdf. Accessed March 23, 2020. 2. Accreditation Council for Pharmacy Education. Accreditation Standards and Key Elements for the Professional Program in Pharmacy Leading to the Doctor of Pharmacy Degree ("Standards 2016"). Published February 2, 2015. https://www.acpe-accredit.org/ pdf/Standards2016FINAL.pdf. Accessed March 23, 2020.

3. American Association of Colleges of Pharmacy 2016 Council of Faculties Business Meeting, Anaheim, July 25, 2016. Am J Pharm Educ. 2016;80(8):Article S11.

4. Rivkin A. Thinking clinically from the beginning: early introduction of the pharmacists' patient care process. Am J Pharm Educ. 2016;80(10):Article 164.

5. Rebitch CB, Fleming VH, Palmer R, Rong H, Choi I. Evaluation of video-enhanced case-based activities guided by the Pharmacists' Patient Care Process. Am J Pharm Educ. 2019;83(4):Article 6676.

6. Gonyeau MJ, DiVall M, Conley MP, Lancaster J. Integration of the Pharmacist's Patient Care Process (PPCP) into a comprehensive disease management course series. Am J Pharm Educ. 2018;82(6): Article 6311.

7. Sease JM, Hamptom T, Eagerton DH. Evaluation of student performance of the Pharmacists' Patient Care Process using simulation IPPE. Am J Pharm Educ. 2017;81(5):Article S5.

8. Caimano CR, Lodise NM, Strang AF, Brewer JM. Integration of the Pharmacists' Patient Care Process at Albany College of Pharmacy and Health Sciences. Am J Pharm Educ. 2017;81(5): Article S5.

9. Porter BL, Sevin AM, Spiers B, Trolli E, McAuley JW. Incorporating the Pharmacists' Patient Care Process in experiential education. AACP Experiential Education Section Newsletter. 2016; 4(1):1-2.

10. Divine H, Taylor S, Kebodeaux C, McIntosh T, Policastri A, Jones M. The perfect PaCE: the Pharmacists' Patient Care Process in an integrated patient care laboratory/experiential education course. AACP Experiential Education Section Newsletter. 2016;4(1):1-2. 11. Cooley J, Lee J. Implementing the Pharmacists' Patient Care Process at a Public Pharmacy School. Am J Pharm Educ. 2018; 82(2):Article 6301.

12. Phillips BB, Newsome AS, Bland CM, et al. Evaluation of student performance and confidence in a pharmacy capstone course utilizing the Pharmacists' Patient Care Process. Am J Pharm Educ. 2019;83(8):Article 7357. www.ajpe.org/doi/pdf/10.5688/ajpe7357. 13. Haines ST, Pittenger AL, Stolte SK et al. Core entrustable professional activities for new pharmacy graduates. Am J Pharm Educ. 2017;81(1):Article S2. 